\title{
Comparative Performance among Hamilton Crosser and Yamada Ota Hybrid Nanofluid Models in Generalized Mass Diffusion and Heat Energy across a Vertical Heated Riga Plate via Finite Element Analysis
}

Fuzhang Wang

Xuzhou University of Technology

Muhammad Sohail ( $\nabla$ muhammad_sohail111@yahoo.com ) Institute of Space Technology

Essam R. El-Zahar

Prince Sattam bin Abdulaziz University

Umar Nazir

Institute of Space Technology

Poom Kumam

King Mongkut's University of Technology Thonburi (KMUTT)

\section{Research Article}

Keywords: Vertical Riga plate, Carreau Yasuda liquid, Electromagnetic force, Hybrid nanoparticles, NonFourier's theory, Chemical reaction

Posted Date: March 3rd, 2022

DOI: https://doi.org/10.21203/rs.3.rs-1375041/v1

License: (c) (i) This work is licensed under a Creative Commons Attribution 4.0 International License. Read Full License 


\section{Comparative Performance among Hamilton Crosser and Yamada Ota Hybrid Nanofluid Models in Generalized Mass Diffusion and Heat Energy across a Vertical Heated Riga Plate via Finite Element Analysis}

Fuzhang Wang ${ }^{1,2}$, Muhammad Sohail ${ }^{3, *}$, Essam R. El-Zahar ${ }^{4,5}$, Umar Nazir ${ }^{3}$ and Poom Kumam ${ }^{6,7, *}$

${ }^{1}$ College of Mathematics and Statistics, Xuzhou University of Technology, 221018 Xuzhou, China

${ }^{2}$ Department of Mathematics, Nanchang Institute of Technology, Nanchang 330044, China;

${ }^{3}$ Department of Applied Mathematics and Statistics, Institute of Space Technology P.O. Box 2750, Islamabad 44000, Pakistan

${ }^{4}$ Department of Mathematics, College of Science and Humanities in Al-Kharj, Prince Sattam bin Abdulaziz University, P.O. Box 83, Al-Kharj 11942, Saudi Arabia

${ }^{5}$ Department of Basic Engineering Science, Faculty of Engineering, Menoufia University, Shebin El-Kom 32511, Egypt

${ }^{6}$ Center of Excellence in Theoretical and Computational Science (TaCS-CoE) \& KMUTT Fixed Point Research Laboratory, Room SCL 802 Fixed Point Laboratory, Science Laboratory Building, Departments of Mathematics, Faculty of Science, King Mongkut's University of Technology Thonburi (KMUTT), 126 Pracha-Uthit Road, Bang Mod, Thung Khru, Bangkok 10140, Thailand

${ }^{7}$ Department of Medical Research, China Medical University Hospital, China Medical University, Taichung 40402, Taiwan

Corresponding Authors: Muhammad Sohail (muhammad sohail111@yahoo.com) and Poom Kumam (poom.kum@kmutt.ac.th)

Abstract: This research considered the utilization of revised heat and mass fluxes to study the mass, heat transport in Carreau Yasuda model past over a vertical Riga plate in the presence of heat generation /absorption and chemical reaction. Thermal and mass transportation also engaged the involvement of variable diffusion coefficient and thermal conductivity. The characteristics of Hamilton crosser and Yamada Ota models for the study of hybrid nanoparticles are discussed. The considered model is developed under boundary layer approach in Cartesian coordinates which results nonlinear coupled PDEs. The derived PDEs has been reduced to corresponding ODEs with the help of an appropriate transformation. Afterwards, transformed ODEs are solved numerically via finite element (FEM) procedure. The authenticity of FEM is shown with the help of grid independent survey and comparative study. The obtained results are in excellent agreement with the previous published data. The utilized approach is effective and highly recommended for the nonlinear problems arising in the modeling of several phenomena.

Keywords: Vertical Riga plate; Carreau Yasuda liquid; Electromagnetic force; Hybrid nanoparticles; Non-Fourier's theory; Chemical reaction. 


\section{Introduction}

Due to the huge practical applications and industrial utilization in different mechanisms the study of nanoparticles and nanofluids got much attraction by the researchers and scientists. The mixture of nanoparticles is an important agent to boost and for the improvement of thermal performance. Several theoretical, numerical and experimental studies have been established to study the inclusion of nanoparticles mixture. For instance, Merabia et al. [1] presented the experimental investigation to monitor the thermal performance with the addition of nanoparticles of different shapes. Madhukesh et al. [2] studied the heat transport in hybrid nanofluid past over a curved elongating sheet. They considered the generalized heat flux for energy transfer. They have solved the resulting nonlinear ODEs numerically and plotted numerous graphs against involved parameter in order to monitor their comportment. They monitor the increase in velocity gradient against curvature parameter. Moreover, heat transfer rate varies directly against thermal relaxation parameter and curvature parameter. Shafiq et al. [3] modelled the viscoelastic second grade fluid with bio-convection phenomenon and with the involvement of chemical reaction for two dimensional flow. They utilized boundary layer theory to develop the model and solved the transformed nonlinear equations numerically. They expressed the solution against numerous parameter in the form of contour plots. They observed the decline in motile density profile against thermophoresis parameter and Pecelt number. Nisar et al. [4] examined the involvement of gold nanoparticles for the micro-polar radiative flow past over a shrinking/stretching curved surface. They computed the solution for governing modeled equation numerically in MATLAB symbolic package. They notice the increase in fluid temperature against radiation parameter. Waqas et al. [5] numerically studied the inclusion of gyrotactic microorganism in modified viscoelastic two dimensional nonlinear flow past over a stretching sheet. They disclosed the rise in concentration field against Prandtl number and reverse behavior is reported for temperature profile. Swain et al [6] studied heat and mass transfer in MHD flow of radiated hybrid nanofluid past over an experimentally stretching sheet. They considered the involvement of chemical reaction; slip conditions and heat generation. They converted the derived PDEs into ODEs with the help of similarity transformation and then approximated the converted ODEs numerically. They notice the decline in fluid concentration against Schmidt number and chemical reaction parameter. Moreover, they presented the comparison for the temperature field for three different 
cases. They recorded the increase in fluid temperature against radiation parameter. Also, they presented that the temperature for hybrid nanofluid is higher than nanofluid which is higher than pure water. Gul and Firdous [7] experimentally presented their survey on dispersion of grapheme nanoparticles to investigate thermal transmission for the flow between rotating disks. They handled the develop nonlinear ODEs via OHAM scheme coded in MATHMATICA 15.0 package. They recorded the decrease in thermal conductivity of nanofluid which varies linearly against flow temperature. Tassaddiq et al. [8] presented the analytical solution for the flow between rotating disk having CNTs mixed particles. They presented the error analysis for used scheme. They observed that due to mixing of nanoparticle in carrier liquid the skin friction coefficient depreciates. Iacovita et al. [9] presented the study on augmentation of nanoparticle which was used to reduce the cells of cancer. Khan et al. [10] studied the hybrid nanofluid flow past over a slendring surface in the presence of radiation effect. They studied the assisting and opposing behavior of velocity graphically against numerous parameter. They found that higher values of slender surface parameter increase the fluid velocity and it is further expressed that dual behavior of velocity is noticed against volume fraction. Biswas and $\mathrm{Wu}$ [11] studied the involvement of nanoparticles on studying the impact of temperature on surroundings. Mohanraj and Chen [12] presented a comprehensive review of utilization of nanoparticles in different mechanism. Win-Shwe and Fujimaki [13] presented experimental survey of nanoparticles utilization in different industrial situations. Do et al. [14] analyzed the biomedical applications of nanoparticles and mentioned their use in different processes. Several important contributions have been made on nanoparticle utilization under different effects and are reported by [15-19] and references therein.

Available studies show that no investigation on hybrid Nano fluid with generalized models in reported over a Riga plate. This contribution fills this gap. This report is organized as: literature survey is mentioned in section 1 , modeling is covered in section 2 , section 3 contains the methodology, solution is discussed in section 4 with physical interpretation, conclusion is reported in section 5 .

\section{Preparation of model}

A vertical Riga plate is considered to visualize thermal and mass species effects in Carreau Yasuda liquid in the presence of mixture of nanoparticles. Electromagnetic force is considered 
along with bouncy forces. Chemical species and heat source are inserted in non-Fourier's law whereas motion into particles is developed with help of movement of wall. Temperature dependent mass diffusion and thermal conductivity are addressed in the presence of hybrid nanoparticles. Yamada Ota hybrid and Hamilton Crosser models are used. A Riga plate and stretching surface are sketched by Fig. 1 is sketched for geometrical behavior of flow situation. Boundary layer approximations are implemented to construct system of PDEs. Hence, a system of PDEs is

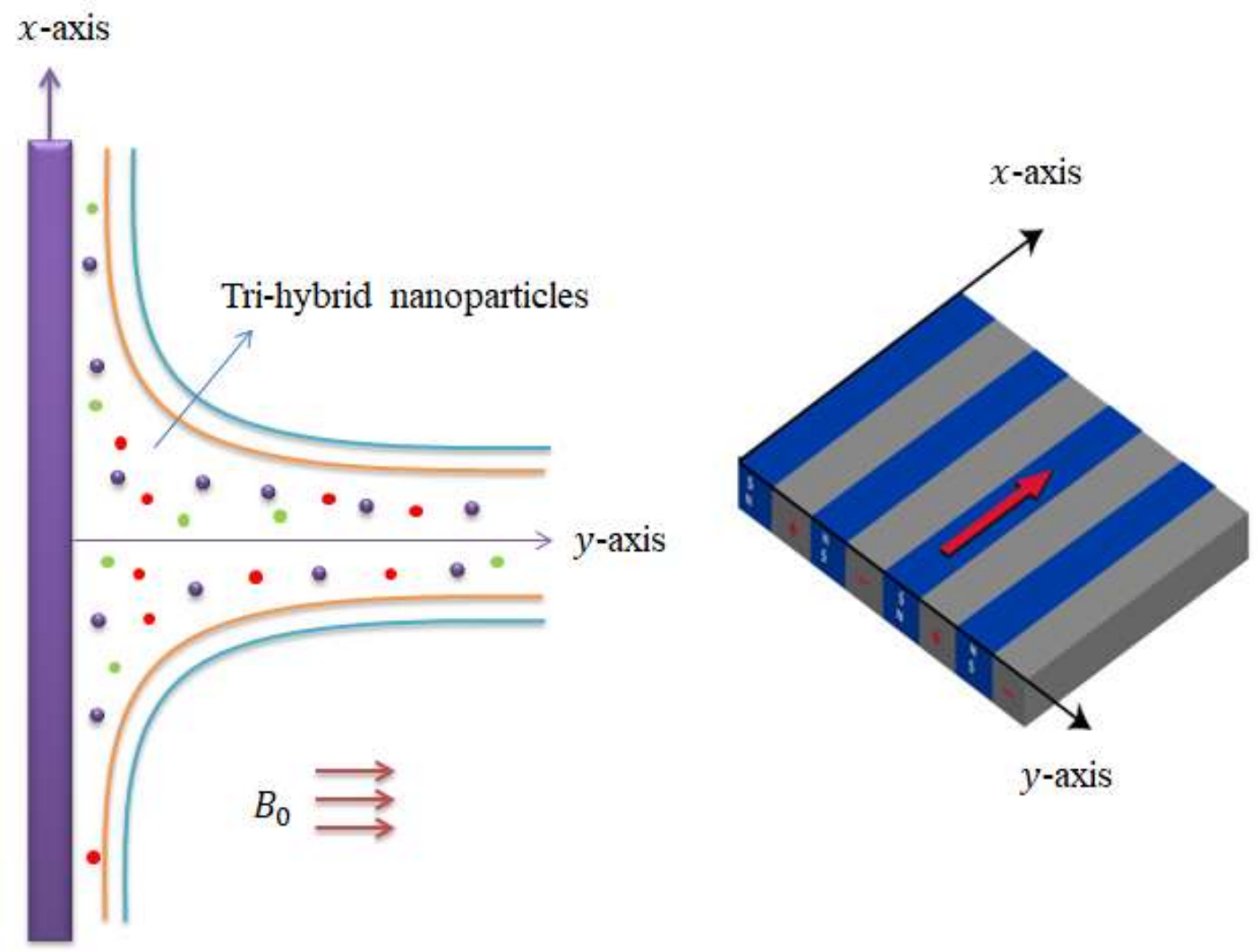

Figure 1. Geometry and coordinates system.

$$
\begin{gathered}
\frac{\partial V_{1}}{\partial x}+\frac{\partial V_{2}}{\partial y}=0, \\
V_{1} \frac{\partial V_{1}}{\partial x}+V_{2} \frac{\partial V_{1}}{\partial y}=\frac{-G \beta_{1}\left(C-C_{\infty}\right)}{\rho_{\text {hybrid }}}-\frac{G \beta_{1}\left(T-T_{\infty}\right)}{\rho_{\text {hybrid }}}+\frac{M_{0} \pi j_{0}}{8 \rho_{\text {hybrid }}} \exp \left(-\frac{\pi}{a} y\right)
\end{gathered}
$$




$$
\begin{gathered}
+v_{\text {hybrid }} \frac{\partial^{2} V_{1}}{\partial y^{2}}+v_{\text {hybrid }} \Gamma^{d}\left(\frac{n-1}{d}\right)(d+1) \frac{\partial^{2} V_{1}}{\partial y^{2}}\left(\frac{\partial V_{1}}{\partial y}\right)^{d}, \\
V_{1} \frac{\partial T}{\partial x}+V_{2} \frac{\partial T}{\partial y}+\gamma_{1}\left[\begin{array}{c}
V_{1}{ }^{2} \frac{\partial^{2} T}{\partial x^{2}}+V_{2}{ }^{2} \frac{\partial^{2} T}{\partial y^{2}}+2 V_{1} V_{2} \frac{\partial T}{\partial x \partial y}+\left(V_{1} \frac{\partial V_{1}}{\partial x}+V_{2} \frac{\partial V_{1}}{\partial x}\right) \frac{\partial T}{\partial x} \\
+\left(V_{1} \frac{\partial V_{2}}{\partial x}+V_{2} \frac{\partial V_{2}}{\partial x}\right) \frac{\partial T}{\partial y}-\frac{Q_{0}}{\left(\rho C_{p}\right)_{h n f}}\left(V_{1} \frac{\partial T}{\partial x}+V_{2} \frac{\partial T}{\partial y}\right)
\end{array}\right] \\
=\frac{1}{\left(\rho C_{p}\right)_{h n f}} \frac{\partial}{\partial y}\left(k_{h y b r i d}^{T} \frac{\partial T}{\partial y}\right)-\frac{Q_{0}}{\left(\rho C_{p}\right)_{h y b r i d}}\left(T-T_{\infty}\right), \\
V_{1} \frac{\partial C}{\partial x}+V_{2} \frac{\partial C}{\partial y}+\gamma_{1}\left[\begin{array}{c}
V_{1}{ }^{2} \frac{\partial^{2} C}{\partial x^{2}}+V_{2}{ }^{2} \frac{\partial^{2} C}{\partial y^{2}}+2 V_{1} V_{2} \frac{\partial C}{\partial x \partial y}+\left(V_{1} \frac{\partial V_{1}}{\partial x}+V_{2} \frac{\partial V_{1}}{\partial x}\right) \frac{\partial C}{\partial x} \\
+\left(V_{1} \frac{\partial V_{2}}{\partial x}+V_{2} \frac{\partial V_{2}}{\partial x}\right) \frac{\partial C}{\partial y}-K_{M}\left(V_{1} \frac{\partial C}{\partial x}+V_{2} \frac{\partial C}{\partial y}\right) \\
=K_{M}\left(C-C_{\infty}\right)+D_{h y b r i d} \frac{\partial}{\partial y}\left(k_{h y b r i d}^{T} \frac{\partial C}{\partial y}\right) .
\end{array}\right]
\end{gathered}
$$

Subjected to BCs

$$
\begin{gathered}
C=C_{w}, a x=V_{1}, V_{2}=0, T=T_{w}, y:=0 \\
C \rightarrow C_{\infty}, V_{1} \rightarrow 0, T \rightarrow T_{\infty}: y \rightarrow \infty,
\end{gathered}
$$

Desired similarity variables are

$$
\eta=y\left(\frac{u_{w}}{x v_{\infty}}\right)^{\frac{1}{2}}, \frac{T-T_{\infty}}{T_{w}-T_{\infty}}=\theta, \phi=\frac{C-C_{\infty}}{C_{w}-C_{\infty}}, V_{1}=a x F^{\prime}, V_{2}=-\sqrt{a v_{f}} F
$$

Temperature dependent mass diffusion and thermal energy are defined as

$$
\begin{gathered}
k_{\text {hybrid }}^{t}=k_{\text {hybrid }}\left[1+\epsilon_{1}\left(\frac{T-T_{\infty}}{T_{w}-T_{\infty}}\right)\right], \\
D_{\text {hybrid }}^{c}=D_{\text {hybrid }}\left[1+\epsilon_{2}\left(\frac{T-T_{\infty}}{T_{w}-T_{\infty}}\right)\right] .
\end{gathered}
$$

A system of ODEs is formulated as

$$
F^{\prime \prime \prime}+\frac{(d+1)(n-1)}{d} W e^{d} F^{\prime \prime \prime}\left(F^{\prime \prime}\right)^{d}+\frac{v_{f}}{v_{\text {hybrid }}}\left(F F^{\prime \prime}-F^{\prime} F^{\prime}\right)+\frac{v_{f}}{v_{\text {hybrid }}} \lambda_{1} \theta+\frac{v_{f}}{v_{\text {hybrid }}} \lambda_{2} \varphi
$$




$$
\begin{gathered}
+\frac{\omega \exp }{A_{1}}(-\eta \beta)=0, \\
\left(1+\epsilon_{1} \theta\right) \theta^{\prime \prime}+\epsilon_{1}\left(\theta^{\prime}\right)^{2}-\beta_{a} P r \frac{k_{f}\left(\rho C_{p}\right)_{h y b r i d}}{k_{\text {hybrid }}\left(\rho C_{p}\right)_{f}}\left(F F^{\prime} \theta^{\prime}+F^{2} \theta^{\prime \prime}+H_{t} F \theta^{\prime}\right)+\frac{k_{f}}{k_{h y b r i d}} \operatorname{Pr}_{t} \theta \\
+\frac{k_{f}\left(\rho C_{p}\right)_{h y b r i d}}{k_{h y b r i d}\left(\rho C_{p}\right)_{f}} \operatorname{Pr} \mathrm{F} \theta^{\prime}=0, \\
\left(1+\epsilon_{2} \varphi\right) \varphi^{\prime \prime}+\epsilon_{2} \theta^{\prime} \varphi^{\prime}-\frac{\beta_{b} S c\left(1-\phi_{1}\right)^{-2.5}}{\left(1-\phi_{2}\right)^{2.5}}\left(F F^{\prime} \varphi^{\prime}+F^{2} \varphi^{\prime \prime}+\Upsilon F \varphi^{\prime}\right)+\frac{S c\left(1-\phi_{1}\right)^{-2.5}}{\left(1-\phi_{2}\right)^{2.5}} \Upsilon \varphi \\
+\frac{S c\left(1-\phi_{1}\right)^{-2.5}}{\left(1-\phi_{2}\right)^{2.5}} \mathrm{~F} \varphi^{\prime}=0,
\end{gathered}
$$

Boundary conditions in view of dimensionless form are

$$
F^{\prime}(\infty)=0, \theta(0)=1, \varphi(0)=1, F(0)=0, F^{\prime}(0)=1, \theta(\infty)=0, \varphi(\infty)=0 .
$$

Hybrid correlations among nanoparticles are delivered as follows and their properties are mentioned in table 1.

$$
\begin{aligned}
& \rho_{\text {hnf }}=\left[\left(1-\phi_{2}\right)\left\{\left(1-\phi_{1}\right) \rho_{f}+\phi_{1} \rho_{s_{1}}\right\}\right]+\phi_{2} \rho_{s_{2}} \\
& \left.\left(\rho C_{p}\right)_{h n f}=\left[\left(1-\phi_{2}\right)\left\{\begin{array}{c}
\left.\left(1-\phi_{1}\right)\left(\rho C_{p}\right)_{f}\right) \\
+\phi_{1}\left(\rho C_{p}\right)_{s_{1}}
\end{array}\right\}\right]+\phi_{1}\left(\rho C_{p}\right)_{s_{2}}\right\} \\
& \left\{\frac{k_{s_{1}}+(n-1) k_{f}-(n-1) \phi_{1}\left(k_{f}-k_{S_{2}}\right)}{k_{s_{1}}+(n-1) k_{f}-\phi_{1}\left(k_{s_{2}}-k_{f}\right)}\right\}=\frac{k_{b f}}{k_{f}} \\
& \mu_{h n f}=\frac{\left(1-\phi_{2}\right)^{2.5} \mu_{f}}{\left(1-\phi_{1}\right)^{2.5}}, \frac{k_{n f}}{k_{f}}=\left\{\frac{k_{s}+(n+1) k_{f}-(n-1) \phi\left(k_{f}-k_{s}\right)}{k_{s}+(n-1) k_{f}+\phi\left(k_{f}-k_{s}\right)}\right\} \\
& \left.\begin{array}{l}
\frac{k_{h n f}}{k_{b f}}=\left\{\frac{k_{s_{2}}+(n-1) k_{b f}-(1-n) \phi_{2}\left(k_{s_{2}}-k_{b f}\right)}{k_{s_{2}}+(n-1) k_{b f}-\phi_{2}\left(k_{b f}-k_{s_{2}}\right)}\right\} \\
\left\{\frac{k_{s_{2}}+(n-1) k_{b f}-(1-n) \phi_{2}\left(k_{s_{2}}-k_{b f}\right)}{k_{s_{2}}+(n-1) k_{b f}-\phi_{2}\left(k_{b f}-k_{s_{2}}\right)}\right\}=\frac{k_{h n f}}{k_{b f}}
\end{array}\right\},
\end{aligned}
$$

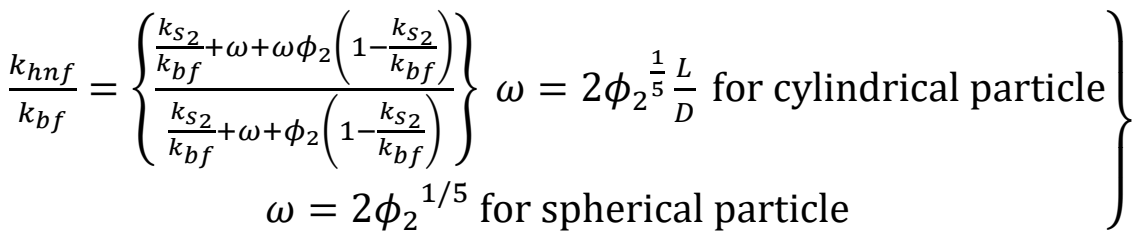




$$
\left.\begin{array}{c}
\frac{k_{h n f}}{k_{b f}}=\left\{\frac{\frac{k_{s_{1}}}{k_{f}}+\omega+\omega \phi_{1}\left(1-\frac{k_{s_{1}}}{k_{f}}\right)}{\frac{k_{s_{1}}}{k_{f}}+\omega+\phi_{1}\left(1-\frac{k_{s_{1}}}{k_{f}}\right)}\right\} \omega=2 \phi_{2}^{\frac{1}{5}} \frac{L}{D} \text { for cylindrical particle } \\
\omega=2 \phi_{2}{ }^{1 / 5} \text { for spherical particles }
\end{array}\right\}
$$

Table 1. Physical properties density, heat capacity and thermal conductivity.

\begin{tabular}{|c|c|c|c|}
\hline & $K$ (thermal conductivity) & $C_{p}$ (heat capicity) & $\rho$ (desity) \\
\hline $\mathrm{C}_{2} \mathrm{H}_{6} \mathrm{O}_{2}$ & 0.253 & 2430 & 1113.5 \\
\hline $\mathrm{SiC}$ & 150 & 1340 & 3370 \\
\hline $\mathrm{TiO}_{2}$ & 8.4 & 692 & 4230 \\
\hline $\mathrm{SiO}_{2}$ & 1.4013 & $3.5 \times 10^{6}$ & 2270 \\
\hline
\end{tabular}

Skin friction coefficient including Carreau Yasuda fluids is

$$
R e^{1 / 2} C f=-\left(1-\phi_{1}\right)^{-2.5}\left(1-\phi_{2}\right)^{-2.5}\left[1+\frac{n-1}{d}\left(W e f^{\prime \prime}(0)\right)^{2}\right] f^{\prime \prime}(0)
$$

Rate of mass diffusion as well as rate of thermal energy transfer is obtained as

$$
\begin{gathered}
N U=\frac{e Q}{\left(T-T_{\infty}\right) k_{\infty}}=-\frac{k_{f}}{k_{h y b r i d} R e^{-\frac{1}{2}}}\left(1+\epsilon_{1}\right) \theta^{\prime}(0), \\
S H=\frac{e m}{\left(C-C_{\infty}\right) D_{\infty}}=-\frac{\left(1-\phi_{2}\right)^{-2.5}}{\left(1-\phi_{1}\right)^{2.5} R e^{-\frac{1}{2}}}\left(1+\epsilon_{2}\right) \varphi^{\prime}(0) .
\end{gathered}
$$

\section{Numerical Procedure and mesh-free analysis}

Weighted residuals: The governing problems are constructed into form of residuals while weight functions are multiplied with desired residuals. So, it gives the form of weighted residuals and this form is integrated over each elements.

Developments of weak forms: The weak form of desired residuals is achieved through process of integration regarding second order terms. Hence, stiffness elements are developed.

Approximations of Galerkin: This useful approximated is used in process of development regarding weak forms whereas element regarding stiffness are achieved. 
Solution of algebraic equations and assembly process: The process of assembly procedure is used for development of system of algebraic equations while this system has been linearized using Picard linearization method. This system is iterative computed.

Mesh free investigation: Convergence analysis of current problem is achieved using mesh free investigation which is performed in table 2. It is observed that problem domain is discretized into three hundred elements to achieve convergence analysis.

Table 2. Grid indepenent analysis of concentration, temperature and velocity at mid of each 300 elements.

\begin{tabular}{|c|c|c|c|}
\hline Number of elements & $f^{\prime}\left(\frac{\eta_{\max }}{2}\right)$ & $\theta\left(\frac{\eta_{\max }}{2}\right)$ & $\varphi\left(\frac{\eta_{\max }}{2}\right)$ \\
\hline 30 & 0.5721974488 & 0.3921033142 & 0.5326877633 \\
\hline 60 & 0.5460428484 & 0.3836649886 & 0.5159889172 \\
\hline 90 & 0.5373843928 & 0.3808296144 & 0.5104234924 \\
\hline 120 & 0.5330677499 & 0.5076407203 & 0.3794064064 \\
\hline 150 & 0.5304818169 & 0.5059713564 & 0.3785500667 \\
\hline 180 & 0.5287599374 & 0.5048579897 & 0.3779786401 \\
\hline 210 & 0.5275304862 & 0.3775697622 & 0.5040635087 \\
\hline 240 & 0.5266088495 & 0.5034653655 & 0.3772636948 \\
\hline 270 & 0.5258925734 & 0.5030028093 & 0.3770243212 \\
\hline 300 & 0.5253200716 & 0.5026336120 & 0.3768329839 \\
\hline
\end{tabular}

\section{Results and Discussion}

Rate of energy transfer and rate of mass diffusion in Carreau Yasuda liquid are measured towards a vertical heated Riga plate. Theory of non-Fourier's law is implemented in the presence of chemical reaction and heat source. Temperature dependent mass diffusion and thermal conductivity are accumulated. Yamada Ota hybrid and Hamilton Crosser models are inserted to enhance thermal energy. Graphical results and numerical tables are tabulated versus various parameters on velocity, concentration and heat energy profiles. Explanations of velocity profile, temperature profile and concentration profile are displayed below.

\subsection{Fluid particles motion and variation in parameters}


Figures 2, 3 and 4 are plotted discussion about velocity profile versus variation in Weissenberg number $(W e)$, heat source parameter $\left(H_{t}\right)$ and $(\omega)$. It is noticed that dot curves are prepared for representation of Yamada-Ota model and dash dot curves are sketched to predict an impact of Hamilton crosser model. Comparative performance among Yamada-Ota model and Hamilton crosser model using variable thermal properties. Figure 2 is prepared to conduct simulations regarding motion of fluid particles versus the variation of We. Mathematically, it is noticed that non-Newtonian model based on Carreau Yasuda fluid is utilized in current flow model. Therefore, an involvement of $W e$ is appeared due to Carreau Yasuda fluid model. It is observed that the motion of particles is slow down when We is increased. Physically, it is noticed that $W e$ is appeared using concept of viscous force and elastic force. Fluid becomes significantly viscous using higher impact of We. Furthermore, curves based on Yamada-Ota model are higher than curves based on Hamilton crosser model. The present model is reduced into Newtonian model when $W e$ is taken as 0 . Flow of fluid particles for case Newtonian model is higher than flow for the case of non-Newtonian model. An involvement of heat source parameter on velocity curves are captured by Fig. 3. Two types of heat source are observed based on heat generation and heat absorption on velocity profile. Negative values of $H_{t}$ are known as heat absorption whereas positive values of $H_{t}$ are called heat generation. Fluid particles absorb more heat energy and heat energy of fluid particles are boosted when heat source parameter is increased. Therefore, motion of fluid particles is boosted versus higher impact of heat source parameter. Moreover, curves for Yamada-Ota model are higher than curves for Hamilton crosser model. Figure 4 reveals behavior of $\omega$ on velocity profile.

\subsection{Thermal energy of fluid particles and variation in parameters}

In this subsection, graphs of thermal energy are plotted against variation in heat source parameter, time relaxation parameter and variable thermal conductivity number. It is noticed that dot curves are prepared for representation of Yamada-Ota model and dash dot curves are sketched to predict an impact of Hamilton Crosser model. Figure 5 is plotted to represent relationship among thermal energy and heat source parameter considering Yamada-Ota and Hamilton crosser hybrid models. It is observed that temperature into fluid particles is boosted when heat source parameter is enhanced. Physically, an external heat source is placed at surface of wall. Hence, temperature of fluid particles is controlled by using positive and negative values 
of heat source parameter. Heat generation phenomenon is occurred when positive values of heat source parameter are used while heat absorption phenomena are happened when negative values of heat source parameter is implemented. Figure 6 is prepared to sketch behavior time relaxation parameter on temperature profile. Time relaxation parameter is generated because of nonFourier's law is utilized in mass diffusion and energy equations. Temperature into fluid particles is enhanced when time relaxation parameter is increased. The capacity of restore of heat energy is enhanced versus argument values of time relaxation parameter. An effect of variable thermal conductivity number on temperature curves is carried out by figure 7 . It is observed that occurrence of $\epsilon_{1}$ is generated using concept of variable thermal conductivity in energy equation. Mathematically, temperature dependent thermal conductivity number is dependent on temperature difference. So, an increment in $\epsilon_{1}$ results heat energy of fluid particles is boosted. Moreover, thermal performance of Yamada-Ota hybrid model is better than thermal performance of Hamilton Crosser hybrid mode.

\subsection{Mass diffusion of fluid particles and variation in parameters}

Figures 8, 9 and 10 are prepared to visualize variation of thermal energy versus change in Schmidt number, variable mass diffusion parameter and time relaxation parameter inserting Yamada-Ota and Hamilton Crosser hybrid models. Mass diffusion performance is measured among Yamada-Ota and Hamilton Crosser hybrid models. Figure 8 is prepared to capture effect of Schmidt number on concentration profile. Diffusion of mass species is slow down using argument numerical values of Schmidt number. From physically point of view, Schmidt number is ratio among mass diffusivity and momentum diffusivity. An inclination in Schmidt number reveals reduction into mass diffusion. Moreover, Performance in mass diffusion rate for Yamada-Ota hybrid model is better than performance in mass diffusion for Hamilton Crosser hybrid model. Role of time relaxation parameter on concentration profile is carried out by figure 9 whereas role of $\epsilon_{2}$ on concentration profile is captured by figure 10. It is investigated that maximum production of mass diffusion is generated when time relaxation and variable mass diffusion parameters. Further, concentration curves for Hamilton Crosser hybrid model are less than concentration curves for Yamada-Ota hybrid model.

\subsection{Measurement of rate of heat transfer, Sherwood number and skin friction coefficient}


In this subsection, performance of temperature gradient, skin friction coefficient and Sherwood number are accumulated versus higher numerical values of Weissenberg, heat source, Schmidt and variable thermal conductivity parameters. The behavior of temperature gradient, skin friction coefficient and Sherwood number versus Weissenberg, heat source, Schmidt and variable thermal conductivity parameters is tabulated in table 3. It is noticed that motion of fluid particles is boosted up against higher numerical values of Weissenberg number but temperature gradient is decreased. In case of heat source parameter, temperature gradient, skin friction coefficient and Sherwood number are augmented considering higher impact of heat source parameter. Sherwood number is inclined when Schmidt number is increased.

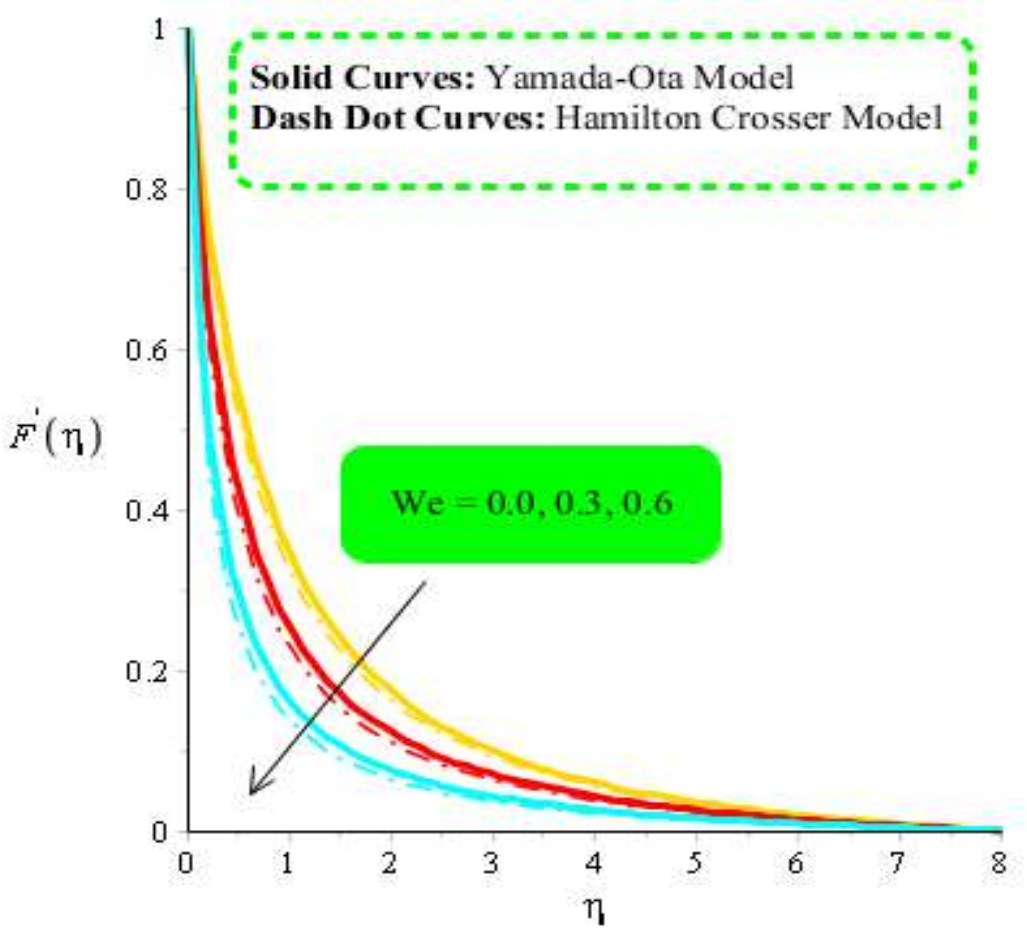

Figure 2. Analysis of $W e$ on velocity regarding fluid particles. 


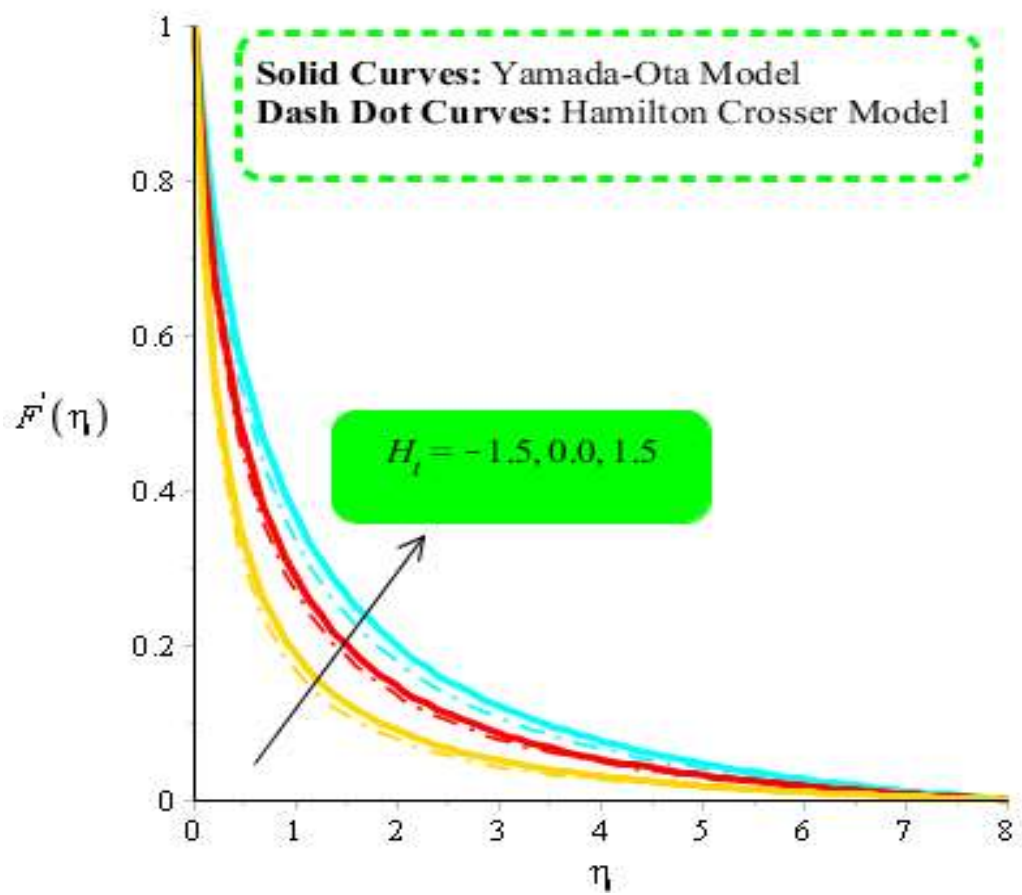

Figure 3. Analysis of $H_{t}$ on velocity regarding fluid particles.

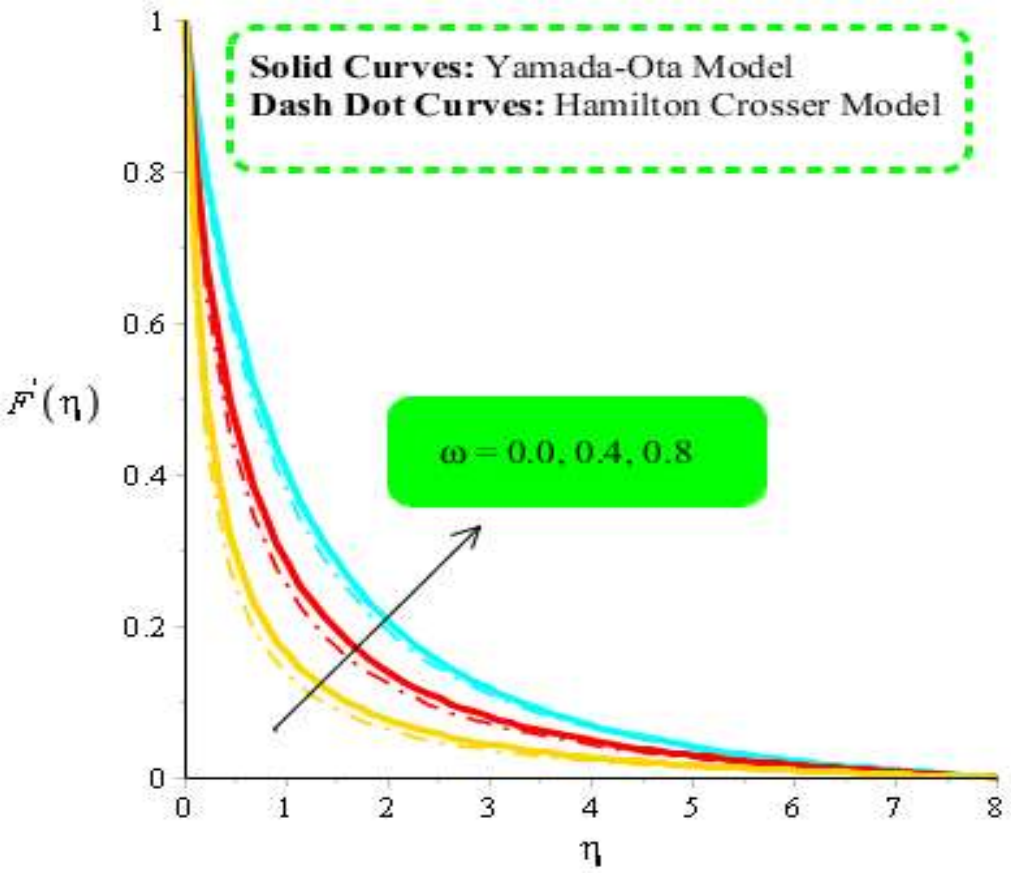

Figure 4. Analysis of $\omega$ on velocity regarding fluid particles. 


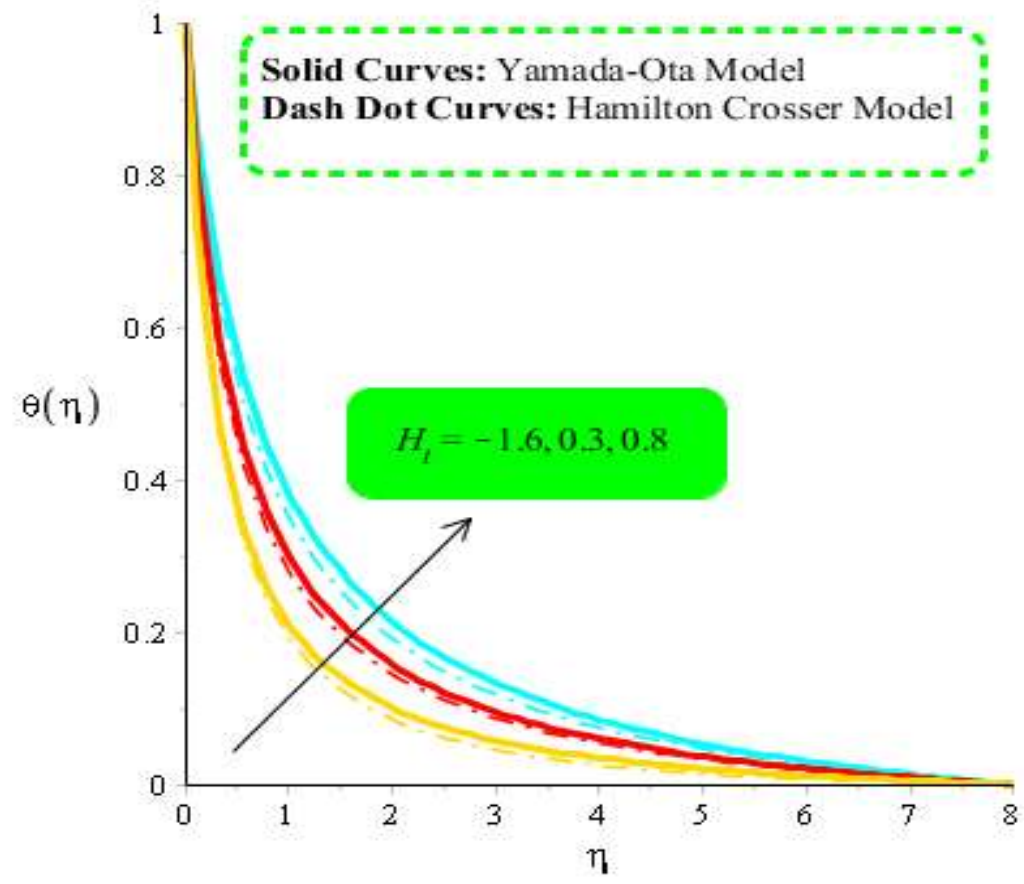

Figure 5. Analysis of $H_{t}$ on temperature regarding fluid particles.

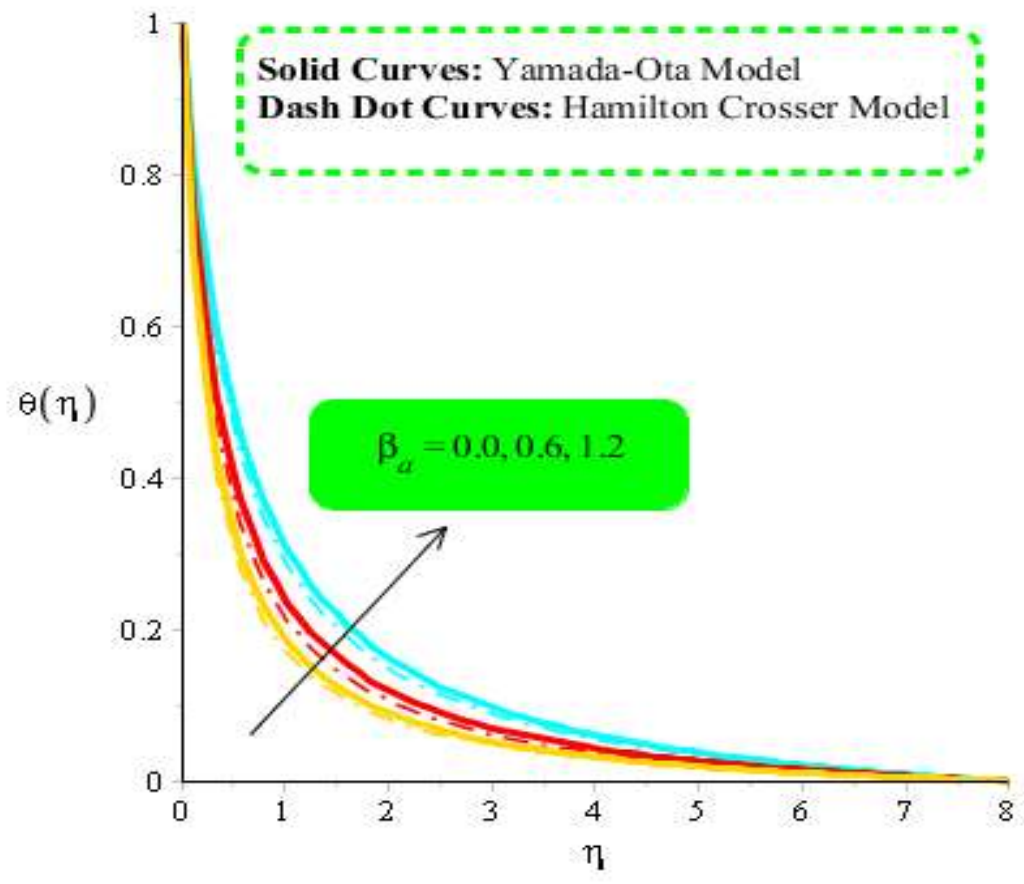

Figure 6. Analysis of $\beta_{a}$ on temperature regarding fluid particles. 


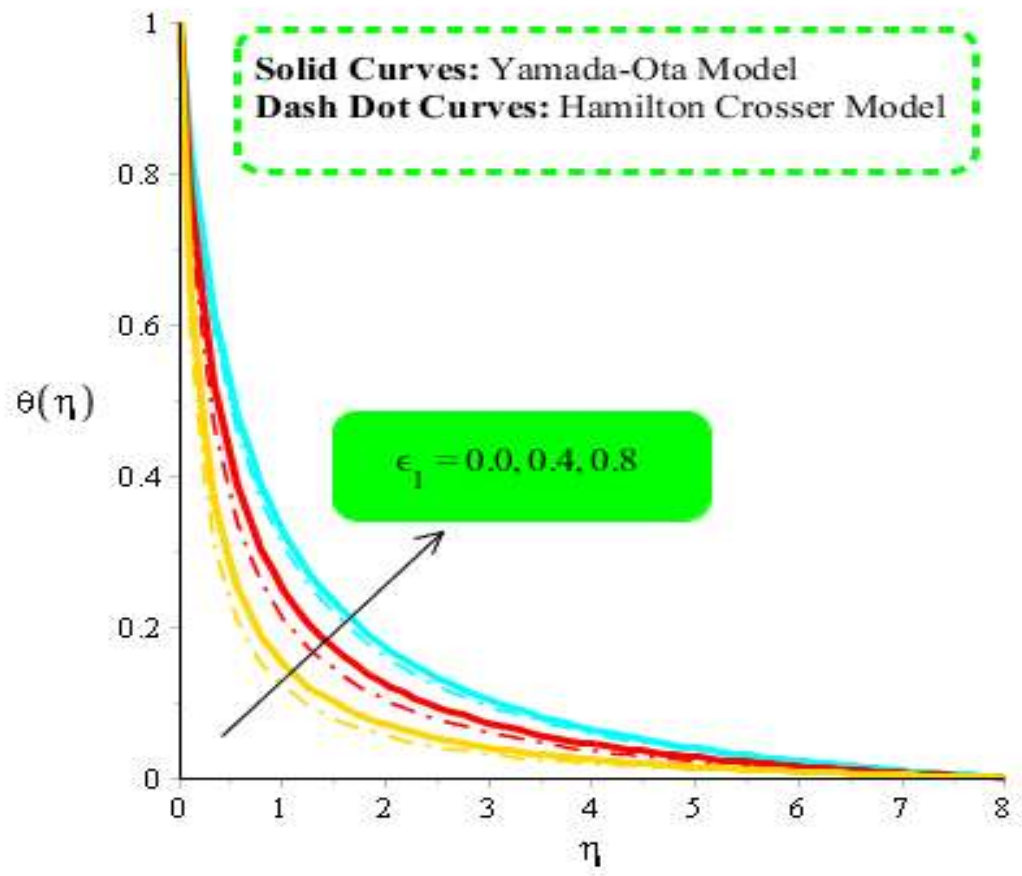

Figure 7. Analysis of $\epsilon_{1}$ on temperature regarding fluid particles.

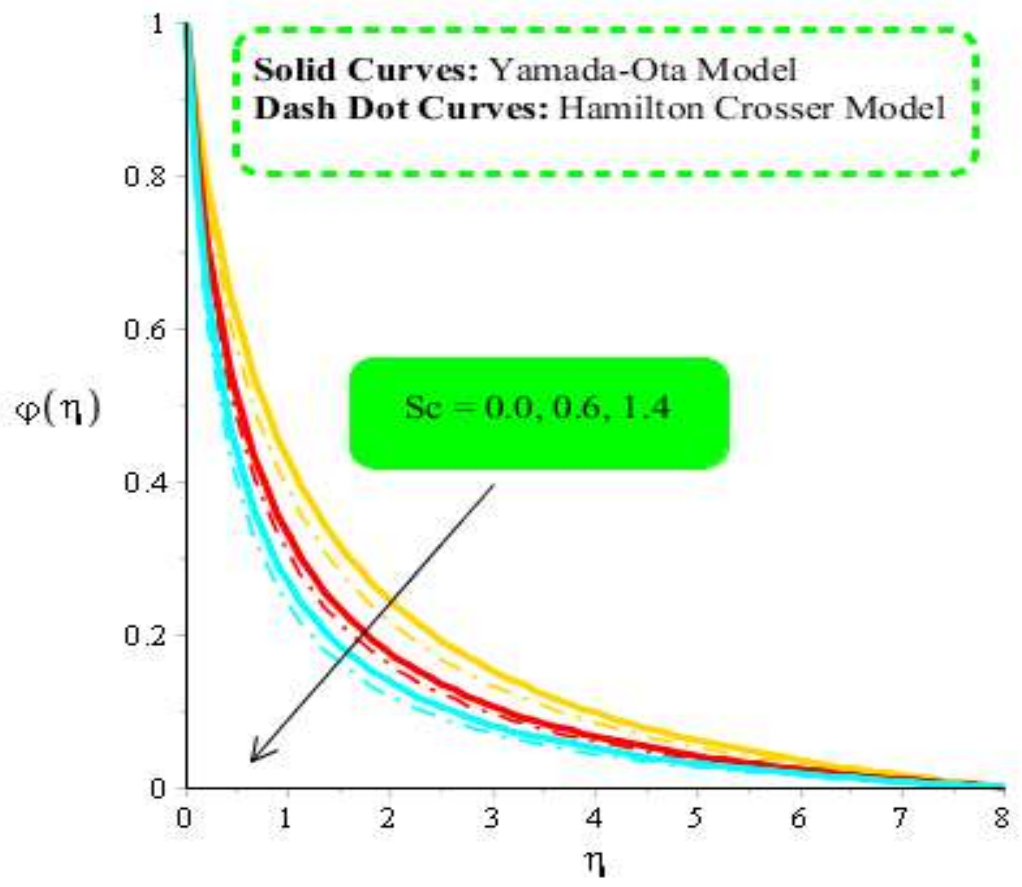

Figure 8. Analysis of $S c$ on concentration regarding fluid particles. 


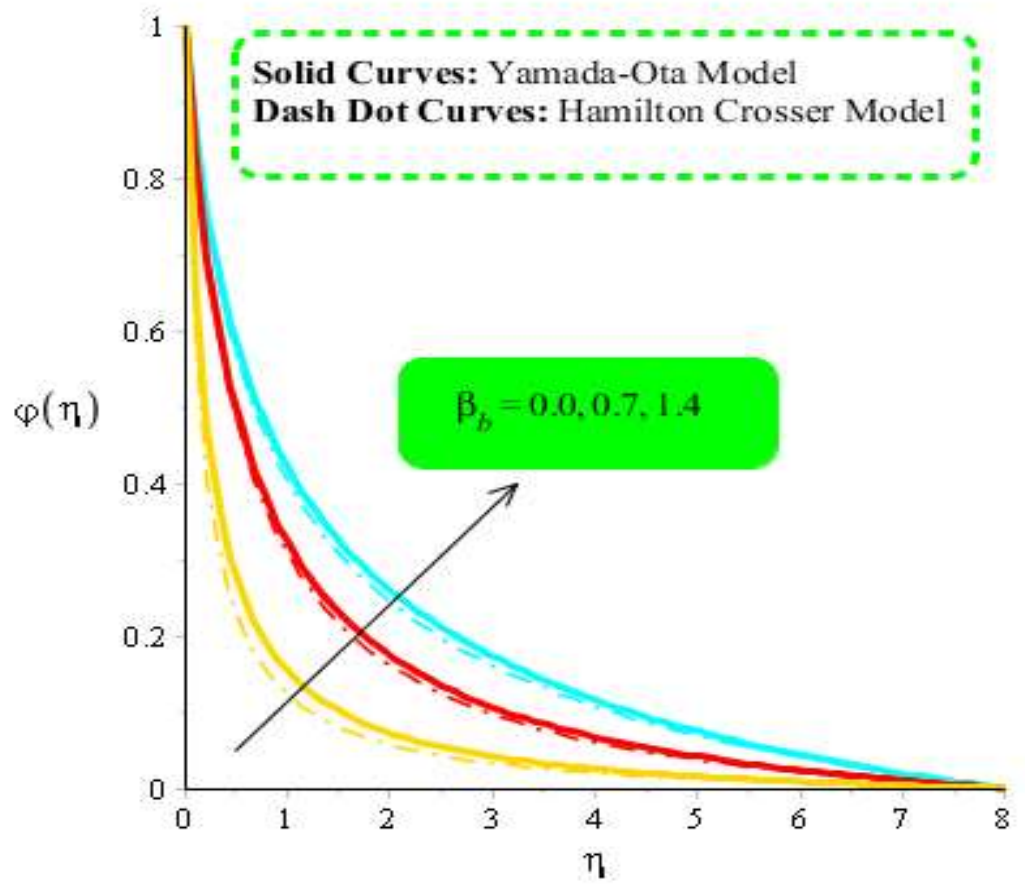

Figure 9. Analysis of $\beta_{b}$ on concentration regarding fluid particles.

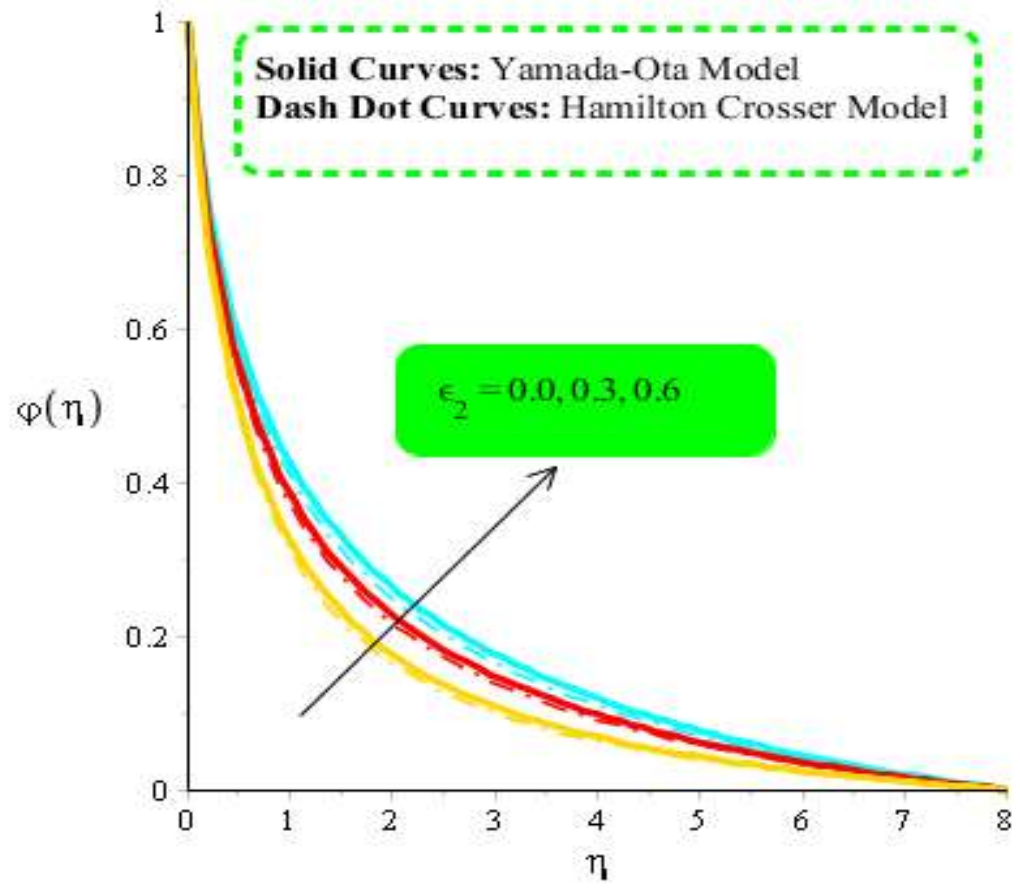

Figure 10. Analysis of $\epsilon_{2}$ on concentration regarding fluid particles. 
Table 3. Analysis of rate of mass diffusion, rate of heat energy and skin friction coefficient against variation in $W e, H_{t}, \epsilon_{1}$ and $S c$.

\begin{tabular}{|c|l|c|c|c|}
\hline $\begin{array}{c}\text { Variation in } \\
\text { parameters }\end{array}$ & & $-R e^{1 / 2} C f$ & $-R e^{-\frac{1}{2}} N U$ & $-R e^{-\frac{1}{2} S h}$ \\
\hline & 0.0 & 0.04083083709 & 0.8718781018 & 0.2251503917 \\
\hline$W e$ & 0.4 & 0.04115641821 & 0.7608320013 & 0.1352215011 \\
\hline & 0.8 & 0.04548199932 & 0.5334280014 & 0.0221003319 \\
\hline & -1.5 & 0.05842070236 & 0.6865965216 & 0.1251639536 \\
\hline$H_{t}$ & 0.0 & 0.02884959667 & 1.023554468 & 0.1310202402 \\
\hline & 0.5 & 0.01075945969 & 1.268425453 & 0.1559011380 \\
\hline$\epsilon_{1}$ & 0.0 & 0.02606740874 & 2.133026234 & 0.7250871596 \\
\hline & 0.3 & 0.03568914202 & 0.687196814 & 0.4512924743 \\
\hline & 0.5 & 0.1784160787 & 0.169312154 & 0.3250943289 \\
\hline & 0.0 & 0.1398472497 & 0.887013278 & 0.3302441317 \\
\hline & 0.4 & 0.2977235842 & 3.131272987 & 0.4135574081 \\
\hline & 0.8 & 0.3536165232 & 5.457159256 & 0.4536907398 \\
\hline
\end{tabular}

\section{Conclusions}

Features of mass diffusion and heat energy in Carreau Yasuda liquid past a vertical heated Riga plate are visualized. Non-Fourier's law is implemented along with temperature dependent mass diffusion and temperature dependent thermal conductivity. Models related to Yamada Ota hybrid and Hamilton Crosser has been carried out. Chemical reaction among mass species particles is occurred. A finite element scheme is utilized to construct numerical simulations. Key findings of present analysis are listed below.

Fluid particles are accelerated when heat source parameter and $\omega$ are increased but fluid particles motion is decreased versus higher values of $W e$; 
$>$ Heat energy of fluid particles is boosted against argument values of heat source, time relaxation and variable thermal conductivity parameters;

$>$ Amount of mass diffusion is declined versus change in Schmidt number but amount of mass diffusion is enhanced versus higher impacts of variable mass diffusion and time relaxation parameters;

> Thermal performance of Yamada-Ota hybrid nanofluid model is better than thermal performance of Hamilton Crosser hybrid nanofluid mode.

Availability of data and material: The data used to support this study are included in the Manuscript.

Conflicts of Interest: The authors declare that they have no conflicts of interest.

Acknowledgments: The authors acknowledge the financial support provided by the Center of Excellence in Theoretical and Computational Science (TaCS-CoE), KMUTT.

Funding: The authors acknowledge the financial support provided by the Center of Excellence in Theoretical and Computational Science (TaCS-CoE), KMUTT.

Authors' contributions: All the authors contributed equally.

\section{References}

[1] Merabia, S., Shenogin, S., Joly, L., Keblinski, P. and Barrat, J.L., 2009. Heat transfer from nanoparticles: A corresponding state analysis. Proceedings of the National Academy of Sciences, 106(36), pp.15113-15118.

[2] Madhukesh, J.K., Kumar, R.N., Gowda, R.P., Prasannakumara, B.C., Ramesh, G.K., Khan, M.I., Khan, S.U. and Chu, Y.M., 2021. Numerical simulation of AA7072AA7075/water-based hybrid nanofluid flow over a curved stretching sheet with Newtonian heating: A non-Fourier heat flux model approach. Journal of Molecular Liquids, 335, p.116103.

[3] Shafiq, A., Rasool, G., Khalique, C.M. and Aslam, S., 2020. Second grade bioconvective nanofluid flow with buoyancy effect and chemical reaction. Symmetry, 12(4), p.621. 
[4] Nisar, K.S., Khan, U., Zaib, A., Khan, I. and Morsy, A., 2020. A novel study of radiative flow involving micropolar nanoliquid from a shrinking/stretching curved surface including blood gold nanoparticles. The European Physical Journal Plus, 135(10), pp.1-19.

[5] Waqas, H., Khan, S.U., Hassan, M., Bhatti, M.M. and Imran, M., 2019. Analysis on the bioconvection flow of modified second-grade nanofluid containing gyrotactic microorganisms and nanoparticles. Journal of Molecular Liquids, 291, p.111231.

[6] Khan, U., Zaib, A., Sheikholeslami, M., Wakif, A. and Baleanu, D., 2020. Mixed convective radiative flow through a slender revolution bodies containing molybdenum-disulfide graphene oxide along with generalized hybrid nanoparticles in porous media. Crystals, 10(9), p.771.

[7] Swain, K., Mebarek-Oudina, F. and Abo-Dahab, S.M., 2022. Influence of MWCNT/Fe3O4 hybrid nanoparticles on an exponentially porous shrinking sheet with chemical reaction and slip boundary conditions. Journal of Thermal Analysis and Calorimetry, 147(2), pp.1561-1570.

[8] Iacoviță, C., Fizeșan, I., Nitica, S., Florea, A., Barbu-Tudoran, L., Dudric, R., Pop, A., Vedeanu, N., Crisan, O., Tetean, R. and Loghin, F., 2021. Silica Coating of Ferromagnetic Iron Oxide Magnetic Nanoparticles Significantly Enhances Their Hyperthermia Performances for Efficiently Inducing Cancer Cells Death In Vitro. Pharmaceutics, 13(12), p.2026.

[9] Gul, T. and Firdous, K., 2018. The experimental study to examine the stable dispersion of the graphene nanoparticles and to look at the $\mathrm{GO}-\mathrm{H} 2 \mathrm{O}$ nanofluid flow between two rotating disks. Applied Nanoscience, 8(7), pp.1711-1727.

[10] Tassaddiq, A., Khan, S., Bilal, M., Gul, T., Mukhtar, S., Shah, Z. and Bonyah, E., 2020. Heat and mass transfer together with hybrid nanofluid flow over a rotating disk. AIP Advances, 10(5), p.055317.

[11] Biswas, P. and Wu, C.Y., 2005. Nanoparticles and the environment. Journal of the air \& waste management association, 55(6), pp.708-746.

[12] Win-Shwe, T.T. and Fujimaki, H., 2011. Nanoparticles and neurotoxicity. International journal of molecular sciences, 12(9), pp.6267-6280. 
[13] Win-Shwe, T.T. and Fujimaki, H., 2011. Nanoparticles and neurotoxicity. International journal of molecular sciences, 12(9), pp.6267-6280.

[14] De, M., Ghosh, P.S. and Rotello, V.M., 2008. Applications of nanoparticles in biology. Advanced Materials, 20(22), pp.4225-4241.

[15] Wang, Fuzhang, Nazir, Umar, Sohail, Muhammad, El-Zahar, Essam R., Park, Choonkil and Thounthong, Phatiphat. "A Galerkin strategy for tri-hybridized mixture in ethylene glycol comprising variable diffusion and thermal conductivity using nonFourier's theory" Nanotechnology Reviews, vol. 11, no. 1, 2022, pp. 834-845.

[16] Hou, E.; Wang, F.; Nazir, U.; Sohail, M.; Jabbar, N.; Thounthong, P. Dynamics of Tri-Hybrid Nanoparticles in the Rheology of Pseudo-Plastic Liquid with Dufour and Soret Effects. Micromachines, 2022, 13, 201. https://doi.org/10.3390/mi13020201

[17] Swain, K., Mebarek-Oudina, F. and Abo-Dahab, S.M., 2022. Influence of MWCNT/Fe3O4 hybrid nanoparticles on an exponentially porous shrinking sheet with chemical reaction and slip boundary conditions. Journal of Thermal Analysis and Calorimetry, 147(2), pp.1561-1570.

[18] Mebarek-Oudina, F., 2019. Convective heat transfer of Titania nanofluids of different base fluids in cylindrical annulus with discrete heat source. Heat TransferAsian Research, 48(1), pp.135-147.

[19] Shah, Z., Khan, A., Khan, W., Alam, M.K., Islam, S., Kumam, P. and Thounthong, P., 2020. Micropolar gold blood nanofluid flow and radiative heat transfer between permeable channels. Computer Methods and Programs in Biomedicine, 186, p.105197. 